\section{Análise dos incentivos contratuais de transplantes de rins no Brasil pelo modelo agente-principal}

\author{
Analysis of contractual incentives for kidney \\ transplants in Brazil using the principal-agent \\ model
}

\author{
Análisis de los incentivos contractuales de trasplantes \\ de riñón en Brasil mediante el modelo \\ agente-principal
}

Cassia Kely Favoretto Costa 1 Giácomo Balbinotto Neto 2,3 Luciano Menezes Bezerra Sampaio 4

\author{
1 Programa de Pós-graduação \\ em Ciências Econômicas, \\ Universidade Estadual de \\ Maringá, Maringá, Brasil. \\ 2 Programa de Pós-graduação \\ em Economia, Universidade \\ Federal do Rio Grande do Sul, \\ Porto Alegre, Brasil. \\ 3 Instituto de Avaliações de \\ Tecnologias em Saúde, \\ Porto Alegre, Brasil. \\ ${ }^{4}$ Departamento de \\ Ciências Administrativas, \\ Universidade Federal do \\ Rio Grande do Norte, \\ Natal, Brasil.
} \author{
C. K. F. Costa \\ Rua Natal 475, Casa B, \\ Maringá, $P R$ \\ 87023-140, Brasil. \\ cfavoretto@hotmail.com
} captadores desse órgão. Os hospitais alocam esforços administrativos, financeiros e gerenciais para realizar as ações de doação, remoção, captaçă custeio de transplantes. A solução para esse tipo de problema está na es truturação de um contrato ótimo de incentivos, no qual se requer um al inhamento de interesses de ambas as partes envolvidas nesse sistema de transplantes.

Transplante de Rim; Economia da Saúde; Gestão em Saúde 


\section{Introdução}

O transplante renal é definido como um procedimento cirúrgico que consiste na transferência deste órgão de um indivíduo (doador vivo e/ou falecido) para outro, objetivando substituir suas funções perdidas $1,2,3$.

O processo de doação-transplante de rim no Brasil é organizado no âmbito do Ministério da Saúde pelo Sistema Nacional de Transplantes (SNT). O órgão doado e captado é distribuído com base em uma lista única de espera (estadual e/ou regional) e sob o consentimento familiar 4 . A participação dos hospitais públicos nessas ações é autorizada e regulada pelo Ministério da Saúde por meio da legislação atual de transplantes 5 , implicando um processo de adesão ${ }^{6}$. Esses hospitais devem também apresentar cadastro atualizado no sistema de Cadastro Nacional de Estabelecimentos de Saúde (CNES) do Sistema Único de Saúde (SUS) 7.

O Brasil apresenta um dos maiores programas públicos de transplantes renais do mundo 8 , sendo responsável por $92 \%$ dos gastos totais nesta área 9. O Ministério da Saúde no SNT financia os valores pagos desde a busca do potencial doador até o monitoramento ambulatorial póstransplante do paciente 10,11 . Os repasses dos recursos financeiros nesse sistema são realizados pelo Fundo de Ações Estratégicas e Compensação (FAEC), que executa o pagamento direto aos estabelecimentos de saúde que atuam no processo de doação-transplante no país 6 .

Destaca-se que além dos pagamentos por procedimentos, o Ministério da Saúde instituiu, por meio da Portaria no 845, de 2 de maio de 2012 12, normas de incentivos financeiros aos hospitais públicos que realizam ações de captação, remoção, transplantes de órgãos e são credenciados no CNES/SUS. O objetivo dessa ação governamental é estimular a quantidade de transplantes de órgãos, reduzir o tempo de espera e melhorar a qualidade de vida dos pacientes após o transplante. Assim, esses sistemas de incentivos tornam-se necessários para garantir melhorias no desempenho do SNT 13.

A atuação dos hospitais públicos e sua tomada de decisão têm influência significativa sobre a eficiência da prestação dos serviços no sistema de transplantes renais 14 . Todo o processo de notificação, captação e distribuição desse órgão é delegado e executado por eles 5 . A oferta de rim, portanto, não é controlada pelos potenciais doadores, mas sim por esses hospitais que decidem se um órgão doado pode ser aproveitado e quem irá recebê-lo, dada a lista única de espera. Além disso, existe a necessidade de um esforço administrativo e organizacional de- les que influencia no número de rins captados para transplante 15,16 .

No período recente, o Brasil apresenta tendência crescente no número de rins transplantados 17. Entre 2004 e 2014, o aumento foi de $73,03 \%$, passando de 3.126 para 5.409 unidades, respectivamente 18 . Contudo, o número de procedimentos realizados está abaixo das necessidades da população, principalmente em razão da falta de doações efetivas desse órgão 19,20. Esse problema pode ocorrer devido a vários fatores, tais como: (i) a baixa remuneração das equipes de saúde responsáveis pelo processo de doação e transplante de órgãos; (ii) a desinformação dos profissionais da saúde; (iii) a dificuldade e o alto custo da manutenção dos potenciais doadores nas Unidades de Terapia Intensiva (UTIs); e (iv) a falta de pessoal treinado para o processo de entrevista familiar, entre outros 20,21 .

O desequilíbrio entre a oferta e a demanda por transplantes renais pode ser decorrente da assimetria de informação (quando uma das partes numa relação contratual tem mais informações relevantes que a outra) existente nos contratos estabelecidos entre os hospitais públicos participantes do SNT e o Ministério da Saúde. Estudos sugerem que essa situação seja analisada por meio do modelo agente-principal 22,23,24.

A abordagem agente-principal diz respeito a uma situação na qual o bem-estar de um indivíduo ou organização ("principal”) depende do esforço e empenho de outro ("agente”) 25,26. O esforço realizado pelo agente não pode ser monitorado e medido pelo principal e, assim, não pode ser diretamente compensado. A solução desse problema está em requerer algum alinhamento de interesses de ambas as partes (agente e principal) 27,28,29,30. Assim, esse modelo ajuda a compreender como são estruturados os contratos de incentivos necessários para lidar com os problemas de incerteza que podem prevalecer nessa área da saúde 31

Nesta pesquisa, os hospitais públicos autorizados pelo SNT a participar das ações de doaçãotransplante de rim podem ser interpretados como agente, e o Ministério da Saúde, o principal. O Ministério da Saúde delega aos hospitais públicos a tarefa de captar rins para transplante, contudo, não observa os esforços de cada um destes hospitais, mas espera o resultado (doações efetivas e transplantes realizados). Em um contexto de informação assimétrica, a estruturação de incentivos e contratos torna-se crucial para as melhorias e aumento da eficiência do SNT.

Na literatura de economia da saúde, diversos autores 32,33,34,35,36,37,38 vêm utilizando o modelo agente-principal na análise das relações contratuais entre equipes médicas e pa- 
cientes, entre as quais, algumas para a área de transplantes 23,24. Todos destacam a existência do problema de assimetria de informação no setor de saúde, especialmente para os transplantes de órgãos. O conhecimento sobre os incentivos contratuais no processo de doação de rim para transplante contribui para reflexões e discussões acerca do tema, proporcionando aos formuladores de políticas públicas subsídios para entender o comportamento dos participantes deste processo e a tomada de decisão para o funcionamento eficiente do SNT 39 .

Diante do exposto, o objetivo do artigo é propor uma análise dos incentivos contratuais para o caso específico dos transplantes renais no Brasil pelo modelo agente-principal. De forma individual, busca-se: (i) descrever o contrato vigente - Portaria no 84512 - entre agente e principal; (ii) identificar a existência (ou não) do problema de risco moral advindo do contrato; e (iii) propor ações de um contrato para aumentar o esforço do agente.

\section{Características econômicas, estruturaçãoe funcionamento do sistema de transplantes de órgãos no Brasil}

No período recente, tem-se desenvolvido um crescente interesse pelas características econômicas do sistema de transplantes de órgãos, as quais podem ser resumidas na Figura 1 40,41. No fluxograma existem oito caixas que abordam os aspectos pesquisados, sendo eles: $\mathrm{A}$ - determinantes do estágio final do órgão doente; $\mathrm{B}$ - medidas de benefícios à saúde; $\mathrm{C}$ - demanda por transplante de órgãos; D - oferta e transplante de órgãos; E - avaliação econômica; F - análise de equilíbrio; $\mathrm{G}$ - avaliação macro; e $\mathrm{H}$ - planejamento, orçamento e monitoramento do transplante de órgãos.

Este artigo concentra-se nos itens C e D, analisando a necessidade do órgão rim para transplante e avaliando os incentivos ofertados aos hospitais públicos que participam das ações de doação-transplante deste órgão (Figura 1).

Em termos econômicos, os órgãos para transplantes apresentam as seguintes características: (i) são essenciais; (ii) a demanda e a oferta são estocástica (ou seja, existe um processo de produção de insumos, devido a uma variação na necessidade e disponibilidade deles); (iii) não podem ser armazenados por muito tempo (com exceção de ossos e medula), sendo usados uma única vez; (iv) têm baixo custo de oportunidade, isto é, em virtude do pouco uso, os órgãos doados se deterioram de forma rápida; (v) têm poucos substitutos (com exceção de rins que admitem os tratamentos dialíticos); (vi) alta capacidade ociosa (elevados desperdícios de órgãos); (vii) a oferta não é controlada pelos doadores; (viii) os candidatos a receptores não comandam a aquisição dos órgãos; e (ix) pode existir um problema de agente-principal, ou seja, de assimetria de informação entre a instituição governamental que delega as ações do processo de doação-transplante e os hospitais responsáveis pelas suas execuções 42,43 .

O Ministério da Saúde, por meio da Coordenação-Geral do Sistema Nacional de Transplantes (CGSNT), atua como a entidade central e articula-se no processo com as instituições estaduais e regionais (Figura 2). A função da CGSNT é regulamentar os procedimentos relativos à captação, alocação e distribuição de órgãos. Ela é apoiada pelos Grupos de Assessoramento Estratégico (GAE), que têm a função de elaborar diretrizes, propor melhorias na legislação, identificar indicadores de qualidade para as atividades de doação e transplante, e emitir pareceres quando solicitados pela CGSNT. As Câmaras Técnicas Nacionais (CTN) também integram o assessoramento à CGSNT e atuam na parte técnica específica desse processo 5 .

A organização do processo logístico e a distribuição de órgãos e tecidos para transplantes são de responsabilidade da Central Nacional de Notificação, Captação e Distribuição de Órgãos (CNNCDO), e executadas nos níveis nacional e estadual pela Central Nacional de Transplantes (CNT) e pelas Centrais de Notificação, Captação e Distribuição de Órgãos (CNCDO), respectivamente (Figura 2) 5.

As Organizações de Procura de Órgãos (OPO) compõem parte do sistema de planejamento, organização e coordenação das doações de órgãos, e sua criação depende das CNCDO de cada estado brasileiro. No aspecto regional, elas atuam em conjunto com as Comissões Intra-Hospitalares de Doação de Órgãos e Tecidos para Transplante (CIHDOTT), realizando a educação continuada nesta área, auxílio aos hospitais para a identificação dos potencias doadores, no processo de diagnóstico de morte encefálica e no encerramento do processo de doação e transplante de órgãos 5 . Assim, baseando-se no funcionamento do SNT é possível compreender o papel do Ministério da Saúde e dos hospitais públicos no modelo agente-principal.

\section{Modelo teórico: agente-principal}

O modelo agente-principal pode ser utilizado para interpretar situações que envolvam a assimetria de informação. O principal, por meio de 


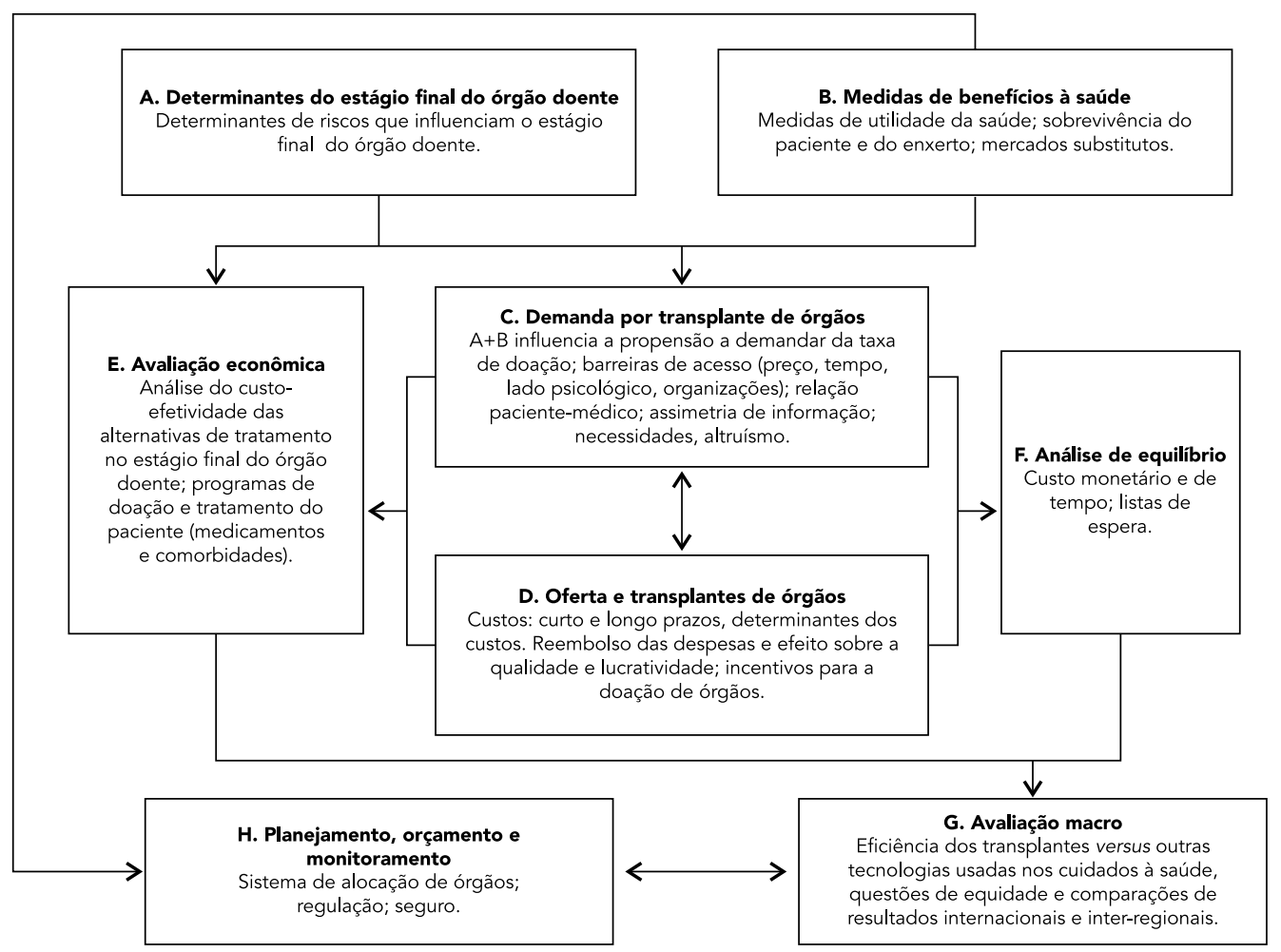

Fonte: adaptado de Williams 40 e Machnicki et al. 41 .

um contrato, delega ações para o agente realizar, e este último decide, se lhe interessa ou não, participar a partir da vantagem de informações que pode dispor 28 .

A informação assimétrica conduz a problemas de controle e gestão denominados seleção adversa (hidden information) e risco moral (hidden actions) 29. O problema de risco moral ocorre depois de ter sido fixado o contrato, ou seja, o principal não consegue monitorar ou observar as ações do Agente. A presença de informação póscontrato conduz a uma perda de bem-estar para os participantes. Já no caso da seleção adversa, as ações do agente são observadas, mas o principal tem dificuldades para avaliá-las 30 .

O modelo agente-principal analisa uma situação na qual o bem-estar de um principal é afetado pelas ações do agente, ou seja, verifica as características da relação contratual e as variáveis que as influenciam de acordo com o comportamento e a informação do agente envolvido $28,29,30$. O contrato ótimo desse método é um documento legal que apresenta de forma objetiva e clara as obrigações dos participantes e as transferências que devem ser realizadas sobre distintas maneiras. As obrigações de cada um dos atuantes são especificadas e incluem os mecanismos de incentivos que o agente receberá para realizar os interesses do principal 25,44. Assim, esse contrato entre as partes envolvidas minimiza os custos e gera o maior resultado possível em termos de produto e divisão de riscos em um contexto de informação assimétrica 26 .

Nessa linha, cada ator tem como objetivo maximizar a sua função de utilidade. $\mathrm{O}$ agente pode não agir de acordo com os interesses do principal. Para esse último alinhar os interesses do agente aos seus, incorre em gastos denominados de custos de agência que correspondem ao somatório: (i) das despesas na elaboração, estruturação e monitoramento do contrato; (ii) dos custos apresentados pelo próprio agente para mostrar ao principal que seus atos lhe serão prejudiciais; e (iii) da perda residual. A perda 
Estrutura e funcionamento do Sistema Nacional de Transplantes (SNT) regulamentado pela Portaria no 2.600 , de 21 de outubro de 2009.

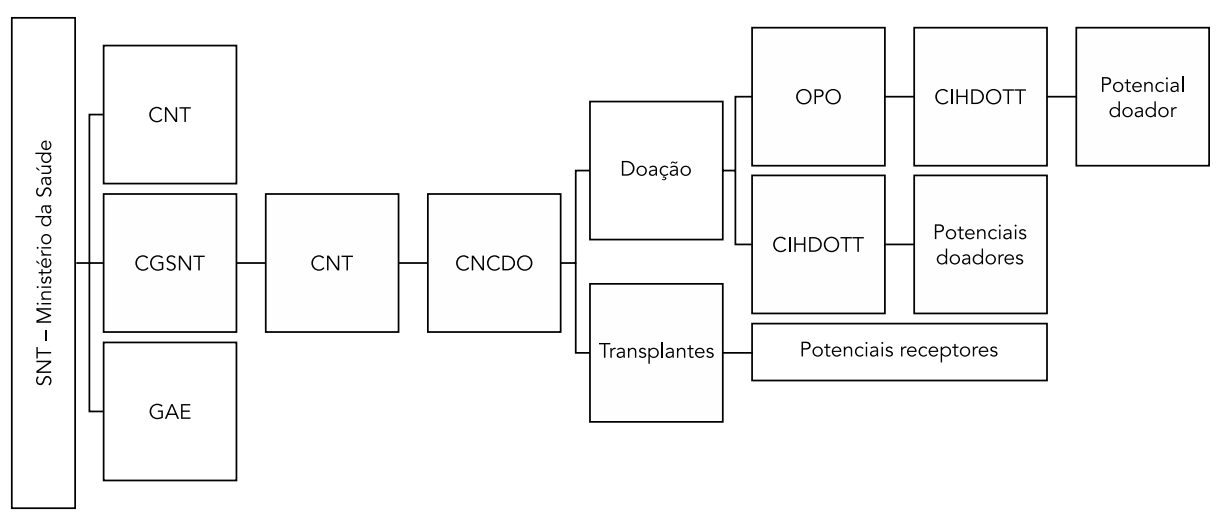

CGSNT: Coordenação-Geral do Sistema Nacional de Transplantes; CIHDOTT: Comissões Intra-Hospitalares de Doação de Órgãos e Tecidos para Transplante; CNCDO: Centrais de Notificação, Captação e Distribuição de Órgãos; CNT: Central Nacional de Transplantes; GAE: Grupos de Assessoramento Estratégico; OPO: Organizações de Procura de Órgãos;

SNT: Sistema Nacional de Transplantes.

Fonte: adaptado de Ministério da Saúde 5

surge da redução dos ganhos do principal por eventuais divergências entre o verdadeiro objetivo do agente e aquele que iria maximizar a riqueza do primeiro ${ }^{28,44}$. O agente necessita ganhar uma recompensa independentemente de qualquer resultado observável, pois apenas terá motivação para realizar o maior esforço se receber um incentivo adicional, e isto gera um custo de agência.

A função de utilidade esperada do principal é assumida ser do tipo Von Neumann-Morgenstern, sendo representada pela equação (1) 28 :

$$
B(x-w)
$$

Na equação (1), as variáveis x e w correspondem ao resultado observável pelo principal e a um custo correspondente aos incentivos que realiza ao agente pelas ações executadas, respectivamente. Essa função não depende diretamente do esforço do agente, mas sim do resultado da tarefa para a qual este foi contratado 29.

A função objetivo do agente, por sua vez, é dada por:

$U(w, e)=u(w)-v(e)$
De acordo com a equação 2, o agente recebe um conjunto de incentivos (w) e realiza um esforço (e) que tem um custo relacionado às ações que lhe foram delegadas. Um maior esforço realizado pelo agente implica maiores custos para o principal, representado pela função de desutilidade $v(e)$. O conjunto de incentivos (w) que o principal oferta ao agente deverá compensá-los por seus esforços adicionais 28,44 .

O principal oferece ao agente um contrato com um período de vigência em que os termos do mesmo não estão sujeitos a uma barganha, ou seja, as alternativas do agente são assumidas neste caso como sendo simplesmente aceitar ou rejeitar o contrato oferecido pelo principal. Nesse contexto, o principal oferece um contrato antecipando o comportamento do agente e busca maximizar sua utilidade, conforme o sistema formado pelas equações 3 , 4 e 5 28,44:

$$
\operatorname{Max}_{\left[e,\left\{w\left(x_{i}\right)\right\}_{i=1, \ldots, n}\right]} \sum_{i=1}^{n} p_{i}(e) B\left(x_{i}-w\left(x_{i}\right)\right)
$$

sujeito a:

$$
\sum_{i=1}^{n} p_{i}(e) u\left(w\left(x_{i}\right)\right)-v(e) \geq \underline{U}
$$

$$
e \in \arg \operatorname{Max}\left\{\sum_{i=1}^{n} p_{i}(\hat{e}) u\left(w\left(x_{i}\right)\right)-v(\hat{e})\right\}
$$


O principal pretende maximizar sua função utilidade esperada (equação 3) e está sujeito, por um lado, pela restrição de participação do agente (equação 4), a qual estabelece que o contrato resulte em uma utilidade para o agente maior que sua utilidade reserva $(\underline{U})$. Essa utilidade representa o valor mínimo que deve induzir o agente a participar do contrato, isto é, o contrato deve permitir-lhe ganhar ou obter uma utilidade esperada superior (ou igual) à de reserva $(\underline{U})$, dado seu esforço (e) 28 . Por outro, o principal deve considerar também a restrição de compatibilidade de incentivos (equação 5), em que o agente deve escolher o nível de esforço compatível com o pagamento que o principal definiu para ele e que também maximize sua utilidade 28,29,44. A notação "arg" representa o conjunto de argumentos da função que maximiza o objetivo do agente, que irá receber o maior incentivo por sua atuação no contrato. Essa equação reflete o problema de risco moral, pois se o agente aceitar o contrato e dado que o seu esforço não é diretamente verificado pelo principal, ele pode eleger o nível de esforço que maximize a sua função objetivo.

Por conveniência analítica, assume-se que o agente escolhe apenas dois níveis de esforço: alto ( $\left.\mathrm{e}^{\mathrm{A}}\right)$ e baixo ( $\left.\mathrm{e}^{\mathrm{B}}\right)$, sendo que $e \in\left\{e^{A}, e^{B}\right\}$. A desutilidade do esforço é mais alta quando o agente emprega um elevado nível de esforço organizacional, ou seja, $v\left(e^{A}\right)>v\left(e^{B}\right)$. Por simplificação, admite-se que: (i) $p_{i}^{A}=p_{i}\left(e^{A}\right)$ e $p_{i}^{B}=p_{i}\left(e^{B}\right)$ para todo $i \in\{1,2, \ldots, n\}$, correspondem à probabilidade de obter-se o resultado $X_{i}$ quando o agente oferta um esforço alto e baixo, respectivamente; e (ii) tais probabilidades são maiores que zero $\left(p_{i}^{A}>0\right.$ e $\left.p_{i}^{B}>0\right) 44$.

No modelo, se o principal demanda que o agente exerça um esforço baixo, não existirá um problema de risco moral; logo, o contrato ótimo seria como no caso de informação simétrica, ou seja, o principal realiza um pagamento ao agente e este irá apresentar um esforço baixo. No entanto, se o principal desejar um alto nível de esforço $\left(e^{A}\right)$ a um baixo $\left(e^{B}\right)$, isto afetará os seus ganhos. Para isso, o principal precisa propor um contrato em que o pagamento dependa do resultado final alcançado. Nesse caso, a equação (5) é reescrita da seguinte forma 44 :

$\sum_{i=1}^{n} p_{i}^{A} u\left(w\left(x_{i}\right)\right)-v\left(e^{A}\right) \geq \sum_{i}^{n} p_{i}^{B} u\left(w\left(x_{i}\right)\right)-v\left(e^{B}\right)$

ou ainda,

$\sum_{i=1}^{n}\left[p_{i}^{A}-p_{i}^{B}\right] u\left(w\left(x_{i}\right)\right) \geq v\left(e^{A}\right)-v\left(e^{B}\right)$

Na equação (6), pode-se interpretar $\sum_{i=1}^{n}\left[p_{i}^{4}-p_{i}^{B}\right] u\left(w\left(x_{i}\right)\right)$ como o bem-estar do agente obtido pela remuneração e v $\left(e^{A}\right)-\mathrm{v}\left(e^{B}\right)$ como o hiato do seu esfor- ço. Os agentes irão escolher o nível de esforço alto $\left(e^{A}\right)$ se o ganho da utilidade esperada com esta ação for superior ao custo implícito de realizá-la (desutilidade) 44 .

O principal deve resolver o seguinte problema implícito de maximização a fim de obter o nível ótimo de esforço do agente 44 :

$\operatorname{Max}_{\left[\left\{w\left(x_{i}\right)\right\}_{i=1, \ldots, n}\right]} \sum_{i=1}^{n} p_{i}^{A}\left[x_{i}-w\left(x_{i}\right)\right]$

sujeito a:

$\sum p_{i}^{A} u\left(w\left(x_{i}\right)\right)-v\left(e^{A}\right) \geq \underline{U}$

$\sum_{i=1}^{n}\left[p_{i}^{A}-p_{i}^{B}\right] u\left(w\left(x_{i}\right)\right) \geq v\left(e^{A}\right)-v\left(e^{B}\right)$

A equação (7) refere-se à função objetivo do principal; a (8) à condição de participação; e a equação (9) trata da condição de compatibilidade de incentivos. No cálculo dos contratos candidatos à solução do problema (7) utiliza-se a regra de Kuhn-Tucker, que é usada em problemas de maximização com restrições de desigualdades. No sistema formado pelas equações (7), (8) e (9) emprega-se o lagrangiano representado por:

$$
\begin{aligned}
L\left(\left\{w\left(x_{i}\right)\right\}, \lambda, \mu\right) & =\sum_{i=1}^{n} p_{i}^{A}\left[x_{i}-w\left(x_{i}\right)\right]+\lambda\left[\sum_{i=1}^{n} p_{i}^{A} u\left(w\left(x_{i}\right)\right)-v\left(e^{A}\right)-\underline{U}\right]+ \\
& +\mu\left[\sum_{i=1}^{n}\left[p_{i}^{A}-p_{i}^{B}\right] u\left(w\left(x_{i}\right)\right)-v\left(e^{A}\right)+v\left(e^{B}\right)\right]
\end{aligned}
$$

Aplicando-se a condição de primeira ordem à equação (10) e considerando que:

$\sum_{i=n}^{n} p_{i}^{A}=\sum_{i=1}^{n} p_{i}^{B}=1$, obtém-se:

$\lambda=\sum_{i=1}^{n} \frac{p_{i}^{A}}{u^{\prime}\left(w\left(x_{i}\right)\right)}>0$

Isso é, a condição de Kuhn-Tucker com relação ao multiplicador da restrição de participação é satisfeita, uma vez que $\lambda \geq 0$. Além disso, essa condição impõe que o multiplicador $(\mu)$ associado com restrição de incentivos deve ser positivo $\mu>044$.

Dividindo-se ambos os lados da equação (11) por $p_{i}^{A}$, pode-se representá-la da seguinte maneira:

$\frac{1}{u^{\prime}\left[w\left(x_{i}\right)\right]}=\lambda+\mu\left[1-\frac{p_{i}^{B}}{p_{i}^{A}}\right]$

sendo $i=1,2, \ldots, n, \lambda>0$ e $\mu>0$

No modelo, a condição $\mu>0$ demonstra que o problema de risco moral gera um custo estritamente positivo para o principal, logo seu bemestar é menor do que em uma situação de informação simétrica 44 .

Na equação (12), a razão de probabilidades $p_{i}^{B} / p_{i}^{A}$ indica que quanto menor a razão, maio- 
res terão de ser os incentivos. Ou ainda, se $p_{i}^{A}$ for superior a $p_{i}^{B}$, maior a certeza de que o sinal do esforço $e^{A}$ é elevado. Portanto, o principal (neutro ao risco) irá oferecer um pagamento ao agente em função do seu resultado e o único propósito desta ação é lhe oferecer incentivos 44 .

\section{Proposta de análise dos incentivos con- tratuais do sistema de transplante renal}

Nesta seção, propõe-se uma análise dos incentivos contratuais do sistema de transplantes renais no Brasil com base na abordagem de agente-principal, conforme antecipado no referencial teórico. O contrato examinado é a Portaria $n_{-}^{o} 84512$, que estabelece estratégia de aumento dos transplantes de órgãos e tecidos no país, por meio da criação de novos procedimentos e de custeio diferenciado para as ações de doaçãotransplante realizadas pelos hospitais que atuam na rede pública de saúde.

A relação de agência entre o Ministério da Saúde e os hospitais públicos pode ser simplificada pela Figura 3. Se o hospital aceitar o contrato proposto pelo Ministério da Saúde, considerando os incentivos que recebe, poderá realizar, por exemplo, dois tipos de esforços: alto (eA) e baixo ( $\left.\mathrm{e}^{\mathrm{B}}\right)$. No primeiro caso, o resultado é um maior número de rins captados (X1); já no segundo, a quantidade é classificada como baixa (X2).

No contrato proposto 12, o Ministério da Saúde busca maximizar o número de doações efetivas e de transplantes renais executados. Para atingir esse objetivo, ele tem um custo que corresponde aos pagamentos dos procedimentos referentes às ações de doação, captação, remoção e transplante do órgão aos hospitais públicos que integram o SNT. O pagamento mensal dessas tarefas é feito pelo FAEC. A verba destinada entra na categoria extrateto (ou seja, valor que ultrapassa a tabela do SUS), pois é direcionada ao custeio de ações de alto custo (transplantes, hemodiálises e medicamentos excepcionais). O FAEC usa os códigos dos procedimentos sobre as Ações Relacionadas à Doação e Transplante de Órgãos, Tecidos e Células, que estão disponíveis no Sistema de Gerenciamento da Tabela de Procedimentos, Medicamentos, Órteses/Próteses e Materiais (OPM) do SUS (SIGTAP. Tabela unificada. http:// sigtap.datasus.gov.br/tabela-unificada/app/sec/ inicio.jsp, acessado em 12/Abr/2015).

Os procedimentos médico-hospitalares dos transplantes de órgãos e tecidos são registrados

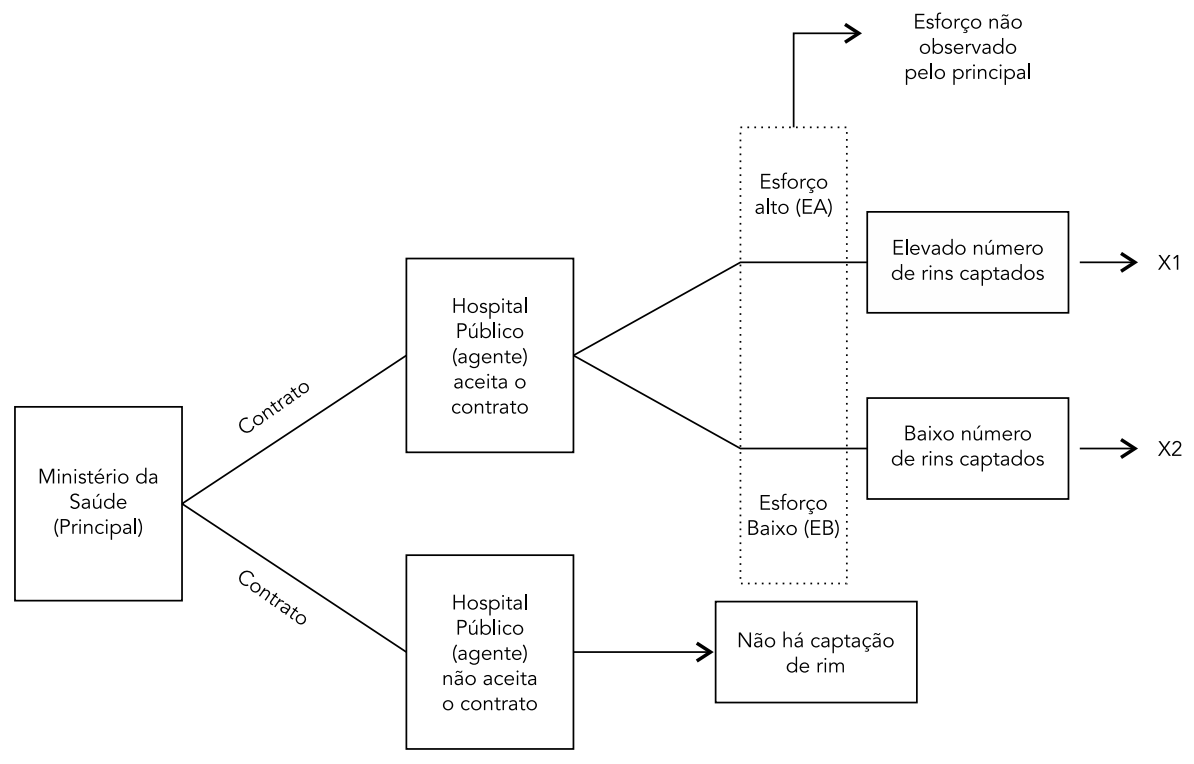

Nota: X1 e X2 são os resultados possíveis obtidos pela captação de rim. 
no Sistema de Informações Hospitalares do SUS (SIH/SUS) por intermédio da Autorização de Internação Hospitalar (AIH). Todos os gestores e prestadores de serviços do SUS usam a AIH como instrumento de registro, que tem como característica a proposta de pagamento por valores fixos desses procedimentos, abrangendo os materiais que devem ser utilizados, as ações que são feitas, os profissionais de saúde envolvidos e a estrutura de hotelaria dos hospitais 6 .

Na Tabela 1 estão os códigos, procedimentos e valores pagos pelo Ministério da Saúde, via FAEC, aos hospitais públicos pelas ações de doação-transplante de rim (Sistema de Gerenciamento da Tabela de Procedimentos, Medicamentos e OPM do SUS. Tabela unificada. http:// sigtap.datasus.gov.br/tabela-unificada/app/sec/ inicio.jsp, acessado em 12/Abr/2015). Os valores unitários em Reais dessas ações são divididos em serviços hospitalares e de profissionais. Para o procedimento de transplante renal-doador falecido, por exemplo, o montante pago é igual a $\mathrm{R}$ \$ 27.622,67, sendo R \$ 19.333,11 referentes aos serviços hospitalares e R \$ 8.289,56 dos profissionais envolvidos no processo (Sistema de Gerenciamento da Tabela de Procedimentos, Medica- mentos e OPM do SUS. Procedimento - transplante de rim: doador falecido. http://sigtap. datasus.gov.br/tabela-unificada/app/sec/proce dimento/exibir/0505020092/04/2015, acessado em 19/Abr/2015). Já para o transplante de rimdoador vivo, o total pago é de $\mathrm{R} \$ 21.238$, 82, composto por $\mathrm{R} \$ 14.865,05$ dos serviços do hospital e $\mathrm{R} \$$ 6.373,77 das equipes de profissionais (Sistema de Gerenciamento da Tabela de Procedimentos, Medicamentos e OPM do SUS. Procedimento transplante de rim: doador vivo. http://sigtap. datasus.gov.br/tabela-unificada/app/sec/proce dimento/exibir/0505020106/04/2015, acessado em 12/Abr/2015).

No contrato proposto 12, o Ministério da Saúde, além dos pagamentos das AIHs, oferece incentivos financeiros aos hospitais públicos que realizam transplante de órgãos e tecidos. Esses incentivos ocorrem por meio do custeio diferenciado Incremento Financeiro para Realização de Procedimentos de Transplantes e o Processo de Doação de Órgãos (IFTDO), que incidem sobre alguns dos valores atuais dos procedimentos relacionados a este processo (Tabela 2), presentes na tabela unificada do SUS (Sistema de Gerenciamento da Tabela de Procedimentos, Medica-

Procedimentos e valores pagos (em Reais) pelo Fundo de Ações Estratégicas e Compensação (FAEC), Ministério da Saúde, pelas ações relacionadas à doação e transplante de rim no Brasil. Sistema de Gerenciamento da Tabela de Procedimentos, Medicamentos e OPM do SUS, competência Abril de 2015.

\begin{tabular}{|c|c|c|c|c|}
\hline Código & Procedimentos & $\begin{array}{c}\text { Serviço } \\
\text { hospitalar } \\
\text { (R\$) }\end{array}$ & $\begin{array}{c}\text { Serviço } \\
\text { profissional } \\
\text { (R\$) }\end{array}$ & Total (R\$) \\
\hline 05.02.01.002-9 & Avaliação clínica de morte encefálica de menor de 2 anos & 125,00 & 150,00 & 275,00 \\
\hline 05.02.01.001-0 & Avaliação clínica de morte encefálica de maior de 2 anos & 75,00 & 140,00 & 215,00 \\
\hline 05.01.06.005-7 & Exame complementar para diagnóstico de morte encefálica & 0,00 & 600,00 & 600,00 \\
\hline $05.01 .07 .003-6$ & Tipagem sanguínea $A B O$ e outros exames hematológicos em possível doador órgãos & 15,00 & 0,00 & 15,00 \\
\hline 05.03.04.005-3 & Entrevista familiar para doação de órgãos de doadores em morte encefálica & 100,00 & 320,00 & 420,00 \\
\hline 05.03.04.006-1 & Entrevista familiar para doação de tecidos de doadores com coração parado & 100,00 & 320,00 & 420,00 \\
\hline 05.03.04.004-5 & Diária de Unidade de Terapia Intensiva de provável doador de órgão & 436,61 & 72,02 & 508,63 \\
\hline 05.03.03.001-5 & Manutenção hemodinâmica de possível doador e taxa de sala para retirada de órgãos & 500,00 & 400,00 & 900,00 \\
\hline 05.06.01.005-8 & Avaliação do possível doador falecido de órgãos ou tecidos para transplante & 0,00 & 215,00 & 215,00 \\
\hline 05.03.03.008-2 & Retirada uni/bilateral de rim (para transplante) & 0,00 & $1.170,00$ & $1.170,00$ \\
\hline 05.03.04.001-0 & Coordenação de sala cirúrgica para retirada de órgãos e tecidos para transplante & 200,00 & 200,00 & 400,00 \\
\hline 05.03.04.002-9 & Deslocamento interestadual de equipe profissional para retirada de órgãos & 0,00 & 900,00 & 900,00 \\
\hline 05.03.04.003-7 & Deslocamento de equipe profissional para retirada de órgãos - intermunicipal & 0,00 & 450,00 & 450,00 \\
\hline 07.02.12.005-7 & Líquido de preservação de rim & 35,00 & 0,00 & 35,00 \\
\hline 05.05.02.009-2 & Transplante de rim (doador falecido) & $19.333,11$ & $8.289,56$ & $27.622,67$ \\
\hline 05.05.02.010-6 & Transplante de rim (doador vivo) & $14.865,05$ & $6.373,77$ & $21.238,82$ \\
\hline
\end{tabular}

Fonte: Sistema de Gerenciamento da Tabela de Procedimentos, Medicamentos e OPM do SUS. (Tabela unificada. http://sigtap.datasus.gov.br/tabelaunificada/app/sec/inicio.jsp, acessado em 12/Abr/2015). 
Tabela 2

Procedimentos e valores (em Reais) sobre os quais incidirá o Incremento Financeiro para Realização de Procedimentos de Transplantes e o Processo de Doação de Órgãos (IFTDO) no processo de doação-transplante renal, conforme Portaria no 845 12, de 2 de maio de 2012 , competência Abril de 2015.

\begin{tabular}{|c|c|c|c|c|}
\hline Código & Procedimentos & Serviço & Serviço & Total (R\$) \\
\hline & & \multicolumn{3}{|c|}{ hospitalar (R\$) profissional (R\$) } \\
\hline 05.03.03.001-5 & Manutenção hemodinâmica de possível doador e taxa de sala para retirada & 500,00 & 400,00 & 900,00 \\
\hline 05.03.03.008-2 & Retirada uni/bilateral de rim (para transplante) & 0,00 & $1.170,00$ & $1.170,00$ \\
\hline \multicolumn{5}{|c|}{ doador falecido } \\
\hline 05.03.04.002-9 & Deslocamento interestadual de equipe profissional para retirada de órgãos & 0,00 & 900,00 & 900,00 \\
\hline 05.03.04.003-7 & Deslocamento de equipe profissional para retirada de órgãos & 0,00 & 450,00 & 450,00 \\
\hline 05.03.04.005-3 & Entrevista familiar para doação de órgãos de doadores em morte encefálica & 100,00 & 320,00 & 420,00 \\
\hline 05.03.04.006-1 & Entrevista familiar para doação de tecidos de doadores com coração & 100,00 & 320,00 & 420,00 \\
\hline 05.03.04.008-8 & Captação de órgão efetivamente transplantado & 260,00 & 0,00 & 260,00 \\
\hline 05.05.02.009-2 & Transplante de rim (órgão de doador falecido) & $19.333,11$ & $8.289,56$ & $27.622,67$ \\
\hline 05.05.02.010-6 & Transplante de rim (órgão de doador vivo) & $14.865,05$ & $6.373,77$ & $21.238,82$ \\
\hline \multirow[t]{2}{*}{$05.06 .02 .005-3$} & Tratamento de intercorrência pós-transplante de rim - pós-transplante crítico & 82,62 & 35,43 & 118,05 \\
\hline & & \multicolumn{2}{|c|}{ Valor ambulatorial } & Total \\
\hline 05.01.05.004-3 & Exames de pacientes em lista de espera para transplante & \multicolumn{2}{|c|}{340,20} & 340,20 \\
\hline
\end{tabular}

Fonte: Sistema de Gerenciamento da Tabela de Procedimentos, Medicamentos e OPM do SUS. (Tabela unificada. http://sigtap.datasus.gov.br/tabelaunificada/app/sec/inicio.jsp, acessado em 12/Abr/2015).

mentos e OPM do SUS. Tabela unificada. http:// sigtap.datasus.gov.br/tabela-unificada/app/sec/ inicio.jsp, acessado em 12/Abr/2015).

Analisando o papel dos hospitais públicos no contrato ${ }^{12}$, supõe-se que a utilidade deles depende de um conjunto de pagamentos (representado pelas AIHs) que eles recebem por cada etapa do processo de doação-transplante de rim. A atuação desses hospitais no SNT pode ocorrer sob três aspectos: (a) ser hospital apenas notificante de morte encefálica; (b) ser notificante, captar o órgão e participar de sua remoção; e (c) realizar a notificação, captação, remoção e transplante do órgão. Os procedimentos e valores pagos pelo Ministério da Saúde, via FAEC, dependem das funções (Tabela 1) que cada um realiza.

Assim, pode-se identificar nesse ponto do contrato 12 a restrição de participação do modelo agente-principal, ou seja, o pagamento por tipo de ações que os hospitais públicos recebem é o valor mínimo (utilidade reserva) para induzi-los a participar do contrato. Com a incidência do IFTDO sobre alguns dos procedimentos (Tabela 2), a utilidade que os hospitais participantes do processo doação-transplante de rim esperam receber pode ser maior que a utilidade reserva, dado o esforço que estão executando neste sistema.

O Ministério da Saúde estabelece no contrato 12 um nível de classificação para os potencial- mente destinatários do IFTDO, conforme segue: (a) os hospitais que realizam quatro ou mais tipos de transplantes de órgãos (nível A) poderão receber um incentivo de $60 \%$ em relação aos procedimentos já pagos no sistema; (b) aqueles que fazem três tipos de transplantes (nível B) receberão o valor de $50 \%$ a mais; (c) as unidades que realizam dois (nível C) ou apenas um tipo (nível D) irão receber IFTDO de $40 \%$ e de $30 \%$ acima do valor, nesta ordem ${ }^{12}$. Nessa situação, o pagamento será mais alto para aqueles hospitais públicos que realizarem o maior número de transplantes renais. Para se enquadrarem nas classificações destacadas, os hospitais deverão realizar esforços no sentido de apresentar ao Ministério da Saúde relatórios com os seguintes indicadores de qualidade: (a) número de transplantes, por órgão, no ano anterior ao do relatório; (b) número de transplantes por milhão de população, por órgão, no ano anterior ao do relatório; (c) curva de sobrevida dos pacientes, por tipo de transplante, no ano anterior ao do relatório; (d) curva de enxertos funcionantes, por tipo de transplante, dos últimos dois anos anteriores ao do relatório; (e) somente será classificado o centro com atividade transplantadora de no mínimo um ano; e (f) os estabelecimentos que realizam transplante de rim deverão apresentar, além dos indicadores destacados, o 
tempo médio decorrido para a confecção das fístulas arteriovenosas pelos serviços de diálises de origem dos pacientes direcionados para transplante, a contar do período do diagnóstico de insuficiência renal crônica 12 .

Nessa etapa do contrato 12 , pode ser evidenciada a restrição de compatibilidade de incentivos do modelo agente-principal, em que os hospitais irão escolher o nível de esforço que é compatível com os pagamentos referentes ao IFTDO. O próprio contrato indica que a diferenciação por nível de esforço entre os hospitais participantes pode ocorrer. De acordo com o modelo de agente-principal 28,44, os hospitais públicos ao aceitarem o contrato vigente e dado o esforço que eles decidem realizar, podem eleger o nível que maximize a função objetivo de cada um. O Ministério da Saúde deseja que os hospitais exerçam os esforços mais altos buscando obter o maior número de transplantes renais, contudo, ele não controla diretamente estas ações. Dessa forma, o Ministério da Saúde, com base nos relatórios comprobatórios emitidos pelos hospitais públicos, pode discriminar o agente mais produtivo dos demais, conceder o incentivo financeiro adequado e, assim, reduzir o risco moral.

Para monitorar e medir os resultados finais dos hospitais públicos no SNT, uma das exigências estabelecidas no contrato pelo Ministério da Saúde é que a classificação deles será renovada a cada dois anos mediante comprovação dos indicadores solicitados. Devido à renovação, o cadastro poderá manter-se igual ou ter o nível alterado, de acordo com os relatórios encaminhados pelos estabelecimentos de saúde 12. Cabe destacar que essa solicitação pode ser inadequada, devido ao fato desse tempo ser demasiadamente longo, particularmente para os hospitais que estão pleiteando passar dos níveis mais baixos de recebimento do IFTDO (C e D) para os mais altos (B e A).

Os hospitais públicos podem ser reclassificados durante o período de vigência das suas classificações atuais no contrato nos seguintes casos: (i) a pedido, mediante aprovação do gestor de saúde estadual e do próprio Ministério da Saúde; (ii) por solicitação de descredenciamento de modalidade de transplantes de órgãos sólidos e/ou de células que definiu a atual classificação; e (iii) se o Ministério da Saúde constatar descumprimento dos requisitos considerados para a classificação. Para reclassificação a pedido, o estabelecimento de saúde deverá encaminhar ao Ministério da Saúde relatórios comprobatórios do enquadramento no nível pretendido, já acompanhados da aprovação do gestor de saúde estadual 12 .

Nesse contexto, considera-se, por hipótese, que esse fato pode gerar uma instabilidade no cadastro do número de estabelecimentos que realizam os transplantes de rim. Se os objetivos mínimos do contrato não forem atingidos, os hospitais podem eleger o nível de esforço baixo e até mesmo deixarem de participar do processo. Nessa situação, o contrato proposto 12 pelo Ministério da Saúde aos hospitais públicos poderá criar incentivos críveis para expandir o esforço dos hospitais na realização dos transplantes de rim.

A solução para esse problema (conforme o modelo agente-principal 28,44) está em apresentar algum tipo de alinhamento de interesses de ambas as partes participantes do contrato (Ministério da Saúde e hospitais públicos). Além disso, o Ministério da Saúde deve continuar empenhando esforços para garantir incentivos financeiros não apenas aos hospitais que realizam os transplantes, mas para aqueles que são notificantes e captadores, bem como para as equipes de transplantes e coordenadores hospitalares 45 , para que tornem uniformes os seus comportamentos e atuem a favor dos objetivos do Ministério da Sáude.

Destaca-se que, além dos incentivos financeiros, existe a necessidade da conjunção de aspectos para que a quantidade efetiva de doações e transplantes renais aumente no Brasil 46. Dentre eles, ressalta-se a necessidade de melhoria das estruturas hospitalares, com recursos físicos e materiais apropriados, bem como recursos humanos mais capacitados e menos sobrecarregados para as funções de identificação de morte encefálica, manutenção do potencial doador de órgãos e abordagem familiar. Além disso, a redução das desigualdades na capacidade operacional das centrais estaduais de transplantes, ativação das comissões intra-hospitalares em estabelecimentos credenciados e melhor execução das regras nacionais em se realizar os exames pré-transplantes pelo SUS 23,45,46.

Portanto, observa-se que a questão dos transplantes renais no Brasil pode ser analisada como um problema de agente-principal. Essa abordagem ao problema fornece uma estrutura teórica sólida e consistente para a formulação de políticas públicas que busquem compreender a questão da assimetria de informação e da estruturação de contratos entre o Ministério da Saúde e os hospitais públicos que atuam no SNT. Ao compreender a relação de agente-principal, é possível minimizar um dos aspectos que pode contribuir para o desequilíbrio entre a oferta e a demanda por rins e, com isto, levar a um aumento de transplantes.

A teoria econômica dos transplantes ainda está em sua infância, não sendo completamente explorada do ponto de vista teórico e empírico, 
e nem de suas implicações para a formulação e implementação de políticas públicas. Essa teoria e abordagem ainda necessita ser mais detalhada e aprofundada. Este artigo é uma pequena, mas original, contribuição para que sejam desenvolvidas futuras pesquisas na área.

\section{Colaboradores}

C. K. F. Costa contribuiu com a concepção do projeto, coleta e interpretação das informações, redação do artigo, correções e aprovação final da versão a ser publicada. G. Balbinotto Neto colaborou na concepção do projeto, análise, correções e aprovação final da versão a ser publicada. L. M. B. Sampaio participou da concepção do projeto, elaboração do artigo, correções e aprovação final da versão a ser publicada.

\section{Agradecimentos}

Ao Conselho Nacional de Desenvolvimento Científico e Tecnológico (CNPq) pela bolsa de produtividade em pesquisa concedida à C. K. F. Costa.

\section{Referências}

1. Khan S, Amedia Jr. CA. Economic burden of chronic kidney disease. J Eval Clin Pract 2008; 14:422-34.

2. World Health Organization. Global glossary of terms and definitions on donation and transplantation. Geneva: World Health Organization; 2009.

3. Associação Brasileira de Transplantes de Órgãos. Manual de transplante renal. São Paulo: Associação Brasileira de Transplantes de Órgãos; 2010.

4. Ministério daSaúde. História do transplante.http:// aplicacao-homologacao.saude.gov.br/saude/trans plante/home/Destaques/historia-transplante (acessado em 08/Mai/2014).

5. Ministério da Saúde. Portaria no 2.601, de 21 de outubro de 2009. Institui, no âmbito do Sistema Nacional de Transplantes, o Plano Nacional de Implantação de Organizações de Procura de Órgãos e Tecidos - OPO. 2009. Diário Oficial da União 2009; 22 out.

6. Coordenação Geral de Sistemas de Informação, Departamento de Regulação, Avaliação e Controle, Secretaria de Atenção à Saúde, Ministério da Saúde. SIH - Sistema de Informação Hospitalar do SUS: manual técnico operacional do sistema. Brasília: Ministério da Saúde; 2012.

7. Ministério da Saúde. Portaria no 511, de 27 de novembro de 2010. Diário Oficial da União 2010; 28 nov.

8. Becker GS, Elias JJ. Cash for kidneys: the case for a market for organs. http://online.wsj.com/news/ articles/SB100014240527023041494045793225600 04817176\#printMode (acessado em 26/Fev/2014).

9. Marinho A, Cardoso SS, Almeida VV. Desigualdade de transplantes de órgãos no Brasil: análise do perfil dos receptores por sexo e raça ou cor. Rio de Janeiro: Instituto de Pesquisa Econômica Aplicada; 2011.

10. Ferraz AS. Os aspectos éticos e a legislação dos transplantes no Brasil. In: Manfro RC, Noronha IL, Silva Filho AP, organizadores. Manual de transplante renal. Porto Alegre: Manole Conteúdo; 2004. p. 364-70. 
11. Marinho A, Cardoso SS, Almeida VV. Disparidade nas filas para transplantes de órgãos nos estados brasileiros. Cad Saúde Pública 2010; 26:786-96.

12. Ministério da Saúde. Portaria no 845, de 2 de maio de 2012. Estabelece estratégia de qualificação e ampliação do acesso aos transplantes de órgãos sólidos e de medula óssea, por meio da criação de novos procedimentos e de custeio diferenciado para a realização de procedimentos de transplantes e processo de doação de órgãos. Diário Oficial da União 2012; 3 mai.

13. Becker GS, Elias JJ. Introducing incentives in the market for live and cadaveric organ donations. J Econ Perspect 2007; 21:3-24.

14. Friele RD, Coppen R, Marquet RL, Gevers JKM. Explaining differences between hospitals in number of organ donors. Am J Transplant 2006; 6:539-43.

15. Marinho A. Um estudo sobre as filas para internações e para transplantes no Sistema Único de Saúde brasileiro. Rio de Janeiro: Instituto de Pesquisa Econômica Aplicada; 2004.

16. Garcia VD. A política de transplantes no Brasil. Rev AMRIGS 2006; 50:313-20.

17. Associação Brasileira de Transplantes de Órgãos. Dimensionamento dos transplantes no Brasil e em cada estado (2006-2013). Registro Brasileiro de Transplantes 2013; XIX:1-3.

18. Ministério da Saúde. Transplantes por categoria: rim. http://portalsaude.saude.gov.br/images/ pdf/2015/marco/23/Transplante-por-categoriarim-u.pdf (acessado em 12/Abr/2015).

19. Garcia VD. Vamos levando... Registro Brasileiro de Transplantes 2011; XVII:3.

20. Associação Brasileira de Transplantes de Órgãos. Sinal vermelho. Registro Brasileiro de Transplantes 2014; XX:3.

21. Abbud Filho M. Desvendando as causas de não efetivação dos potenciais doadores de órgãos: educar para não punir... quem? Jornal Brasileiro de Transplantes 2006; 9:467.

22. Marinho A. Um estudo sobre as filas para transplantes no Sistema Único de Saúde brasileiro. Cad Saúde Pública 2006; 22:2229-39.

23. Garcia VD, Garcia RL. Avaliação do sistema de transplantes renais no Estado do Rio Grande do Sul/Brasil: uma abordagem de Agente-Principal. Medwave 2010; 10:e4712.

24. Costa CKF. Ensaios sobre a economia dos transplantes renais no Brasil: incentivos e eficiência [Tese de Doutorado]. Porto Alegre: Faculdade de Ciências Econômicas, Universidade Federal do Rio Grande do Sul; 2012.

25. Jensen MC, Meckling WH. Theory of the firm: managerial behaviour, agency costs, and ownership structure. J Financ Econ 1976; 3:305-60.

26. Pratt JW, Zeckhauser RS. Principal and agents: the structure of business. Bringhton: Harvard Business School Press; 1985.

27. Pindhyck RS, Rubinfeld DL. Microeconomia. 7a Ed. São Paulo: Pearson Education do Brasil; 2010.

28. Mas-Colell A, Whiston M, Green JR. Microeconomic theory. Oxford: Oxford University Press; 1995.

29. Araújo F. Teoria econômica do contrato. Coimbra: Almedina; 2007

30. Salanié B. The economics of contracts. Cambridge: MIT Press; 1997.
31. Folland S, Goodman AC, Stano M. Economia da saúde. 5a Ed. Porto Alegre: Bookman/Editora Artmed; 2008.

32. Thompson CR, McKee M. An analysis of hospital capital planning and financing in three European countries: using the principal-agent approach to identify the potential for economic problems. Health Policy 2011; 99:158-66.

33. Lundbäck M. Imperfect agency and the regulation of hospitals. The Geneva Papers on Risk and Insurance Theorys 1997; 22:151-68.

34. Smith PC. Measuring health system performance. Eur J Health Econ 2002; 3:145-8.

35. Langer A, Schröder-Bäck P, Brink A, Eurich J. The agency problem and medical acting: an example of applying economic theory to medical ethics. Med Health Care Philos 2009; 12:99-108. 36.

Ludwig M, Merode FV, Groot W. Principal agent relationships and the efficiency of hospitals. Eur J Health Econ 2010; 11:291-304.

37. Brinkerhoff DW, Bossert TJ. Health governance: principal-agent linkages and health system strengthening. Health Policy Plan 2014; 29:685-93.

38. Polaris JJ. Principles over principals? How innovation affects the agency relationship in medical and legal practice. Yale J Health Policy Law Ethics 2014; 14:296-349.

39. Costa CKF, Balbinotto Neto G, Sampaio LMB. Eficiência dos estados brasileiros e do Distrito Federal no sistema público de transplante renal: uma análise usando método DEA (Análise Envoltória de Dados) e índice de Malmquist. Cad Saúde Pública 2014; 30:1667-79.

40. Williams A. Health economics: the cheerful face of the dismal science? In: Williams AH, editor. Health and economics. London: British Association for the Advancement of Science; 1987. p. 1-11.

41. Machnicki G, Seriai L, Schnitzler MA. Economics of transplantation: a review of the literature. Transplant Rev 2006; 20:61-75.

42. Marinho A, Cardoso SS, Almeida VV. Os transplantes de órgãos nos estados brasileiros. Rio de Janeiro: Instituto de Pesquisa Econômica Aplicada; 2007.

43. Balbinotto Neto G, Silva EM, Campelo AK. The impact of presumed consent law on organ donation: an empirical application from quantile regression for panel data. Alessandria: Azienda Ospedaliera Nazionale SS. Antonio e Biagio e Cesare Arrigo; 2012. (Working Paper of Public Health, 3).

44. Macho-Stradler I, Pérez-Castrillo D. An introduction to the economics of information: incentives and contracts. Oxford: Oxford University Press; 1997.

45. Freire ILS, Vasconcelos QLDAQ, Melo GSM, Torres GV, Araújo EC, Miranda FAN. Facilitadores e barreiras na efetividade da doação de órgãos e tecidos. Texto \& Contexto Enferm 2014; 23:925-34.

46. Pessoa JLE, Schirmer J, Roza BA. Avaliação das causas de recursa familiar à doação de órgãos e tecidos. Acta Paul Enferm 2014; 26:323-30. 


\section{Abstract}

The aim of this article was to analyze contractual incentives for kidney transplants in Brazil based on the principal-agent model. The approach assumes that the Brazilian Ministry of Health is the principal and the public hospitals accredited by the National Transplant System are the agent. The Ministry of Health's welfare depends on measures taken by hospitals in kidney uptake. Hospitals allocate administrative, financial, and management efforts to conduct measures in kidney donation, removal, uptake, and transplantation. Hospitals may choose the levels of effort that are consistent with the payments and incentives received in relation to transplantation costs. The solution to this type of problem lies in structuring an optimal in centives contract, which requires aligning the interests of both parties involved in the transplantation system.

Kidney Transplantation; Health Economics; Health Management

\section{Resumen}

El objetivo del artículo fue analizar los incentivos contractuales de trasplantes renales en Brasil, a partir del modelo agente-principal. Este enfoque asume que el Ministerio de Salud sea el principal y los hospitales públicos, autorizados por el Sistema Nacional de Trasplantes, sean los agentes. El bienestar del Ministerio de Salud depende de las acciones tomadas por los hospitales receptores de este órgano. Los hospitales proporcionan los esfuerzos administrativos, financieros y de gestión para realizar las acciones de donación, extirpación, recepción y trasplante de riñón. Los hospitales pueden escoger los niveles de esfuerzos que son compatibles con los pagos e incentivos recibidos, referentes al costeo de trasplantes. La solución para este tipo de problema está en la estructuración de un contrato óptimo de incentivos, en el que se requiera un alineamiento de intereses de ambas partes involucradas en este sistema de trasplantes.

Trasplante de Riñón; Economía de la Salud; Gestión en Salud 